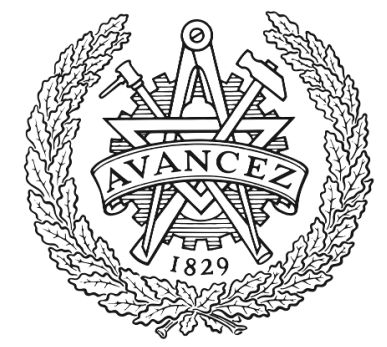

CHALMERS

UNIVERSITY OF TECHNOLOGY

\title{
An STM - SEM setup for characterizing photon and electron induced effects in single photovoltaic nanowires
}

Downloaded from: https://research.chalmers.se, 2023-04-26 08:01 UTC

Citation for the original published paper (version of record):

Holmér, J., Zeng, L., Kanne, T. et al (2018). An STM - SEM setup for characterizing photon and electron induced effects in single photovoltaic nanowires. Nano Energy, 53: 175-181. http://dx.doi.org/10.1016/j.nanoen.2018.08.037

N.B. When citing this work, cite the original published paper. 


\title{
An STM - SEM Setup for Characterizing Photon and Electron Induced Effects in Single Photovoltaic Nanowires
}

\author{
Jonatan Holmér ${ }^{1}$, Lunjie Zeng ${ }^{1 *}$, Thomas Kanne ${ }^{2}$, Peter Krogstrup ${ }^{2}$, Jesper \\ Nygård $^{2}$, Ludvig de Knoop ${ }^{1}$, Eva Olsson ${ }^{1 *}$ \\ ${ }^{1}$ Department of Physics, Chalmers University of Technology, 41296, Göteborg, Sweden. \\ ${ }^{2}$ Center for Quantum Devices and Station Q Copenhagen, Niels Bohr Institute, University of \\ Copenhagen, Universitetsparken 5, 2100 Copenhagen, Denmark.
}

*Corresponding authors: Lunjie Zeng (lunjie@chalmers.se) and Eva Olsson (eva.olsson@chalmers.se)

Keywords: STM - SEM; nanowire-based solar cells; EBIC; GaAs; radial p-i-n junction

\begin{abstract}
Vertical arrays of semiconductor nanowires show great potential for material-efficient and high-performance solar cells. The characterization and correlation between material structure and properties of the individual nanowires are crucial for the continued performance improvement of such devices. In this work, we use a scanning tunneling microscope (STM) probe inside a scanning electron microscope (SEM) to study single photovoltaic nanowires. The STM probe is used to contact individual nanowires in ensembles. We combine the STM-SEM with an in situ light emitting diode (LED) illumination source to study both the electrical and photovoltaic properties of vertical GaAs nanowires with radial p-i-n junctions. We also study the local charge separation ability within the nanowires by electron beam induced current (EBIC) measurements. The in situ SEM setup allows the correlation between properties and nanowire structure. The data show that the quality of the electrical contact to the semiconductor nanowire is crucial to be able to investigate the inherent properties of the nanowires. $\mathrm{We}$ have established a procedure to make high-quality Ohmic contacts to the nanowires with the STM probe. We also show that the effect of mechanical strain on the electrical properties can be investigated by this technique.
\end{abstract}

\section{Introduction}

Semiconductor nanowires have electrical and optical properties that show great potential for photovoltaics [1], [2], [3], [4]. As a result, solar cells composed of arrays of vertical semiconductor nanowires have been the subject of extensive research the past decade. Recently, the record efficiency reached $17.8 \%$ for such a device [5]. The vertical nanowire 
array design offers several advantages compared to thin-film solar cells. First of all, the nanowires have diameters of a few hundred nanometers, which is smaller than the wavelength of visible light. This gives rise to waveguiding and resonance effects [6], [7], making the absorption cross section of a nanowire several times larger than its geometrical cross section [8],[9]. This means that if the nanowire diameter and length, as well as the distance between the nanowires are optimized [10], basically all the incoming light can be absorbed even though the nanowires do not fill up the whole volume of the cell. The material consumption is therefore significantly lower than for a corresponding thin-film solar cell [11]. Additionally, the difference between absorption and geometrical cross section of the nanowires results in a built-in light concentration, which increases the maximum theoretical efficiency of the solar cell [12]. Nanowire arrays also exhibit an anti-reflection property, making anti-reflection coatings superfluous [11]. Furthermore, strain relaxation in the nanowires enables growth of lattice mismatched heterostructures [13] that would not be possible in bulk. It also broadens the choice of possible growth substrates significantly, including inexpensive alternatives as $\mathrm{Si}$ [14]. The nanowire geometry also provides the possibility to separate the direction of light absorption and charge carrier separation by the use of radial $p-n$ junctions. This means that the path for light absorption is long while the distance to the junction separating the electrons and holes is short, which have the potential of optimizing the absorption of light and minimizing the probability for recombination and therefore increase the efficiency [15].

In order to further evolve this relatively new technology, rigorous characterization methods of nanowire solar cell systems are needed. Current-voltage (I-V) measurements of nanowire ensembles in both dark and illuminated conditions can be performed by using standard solar cell characterization techniques, and are important for providing information about the final performance and efficiency of full-scale devices [16]. However, the results of such measurements do not reveal the contribution of each single nanowire. Measurements on 
single nanowires can provide insight into the photovoltaic and electric properties inherent to the nanowires, and how the physical structure of the nanowires affects these properties. This information is important for designing the nanowire solar cells with the best performance. A common strategy to study single nanowires is to lift a wire off the growth substrate and place it horizontally on another substrate, with metal contacts deposited on both ends of the wire [17], [18]. This method works even for radial junction nanowires if one end is etched to reveal the core to the metal contact [19], [20], [21]. This type of measurements can provide useful information about some properties of the nanowires, for example, the minority carrier diffusion length [17]. However, the waveguiding effect is significantly less pronounced in nanowires lying on a substrate compared to vertically standing nanowires [22]. Moreover, if the nanowire has been removed from the growth substrate the effects of the nanowiresubstrate interface cannot be studied. Hence, measurements performed on single vertical nanowires standing on the growth substrate are important for determining the true performance of individual nanowires in complete solar cell devices. A technique for performing measurements on a standing nanowire is reported in [9]. Here, a solar cell device containing only one single vertical nanowire contacted at the bottom via the growth substrate and at the top via a layer of indium tin oxide was fabricated. The result of this work showed, among other things, that the nanowire had the remarkable property of absorbing light from an area approximately 8 times larger than its cross-sectional area. While this characterization technique may provide excellent information about the properties of one single nanowire, it demands the dedicated growth of an individual nanowire solar cell and does not offer the possibility to compare different nanowires in a nanowire ensemble. Recently, characterization of individual as-grown nanowire solar cells have been realized by contacting single nanowires with a nanoprobe, either inside a scanning electron microscope (SEM) [23]or in atomic force microscopy (AFM) [24]. Both these methods enable the probing of individual nanowires in an 
ensemble. However, a remaining challenge is to simultaneously achieve reproducible Ohmic contacts to the nanowires while having the possibility to illuminate the nanowires and study mechanical strain-induced effects.

In this work, we present a technique where a scanning tunneling microscope (STM) probe inside an SEM is used to characterize single as-grown photovoltaic nanowires. Our approach constitutes a method where electrical, photovoltaic and strain-induced properties of individual as-grown semiconductor nanowires for solar cells can be studied simultaneously, with in situ high resolution imaging of the structures. The combination of STM and electron microscopy for making electrical measurements on nanostructures was introduced already around 15 years ago [25], [26] and the technique has been widely used since to investigate, for example, the resistivity of semiconductor nanowires [27], [28], and how the electrical transport properties depend on the diameter [29], mechanical stress [30], [31] and doping profile [30], [32]. Since we are focusing on nanowires for solar cells we are interested in the optical properties and light-induced effects. We have therefore introduced a light emitting diode in the SEM for illumination of the nanowires. We have also established a procedure to control and optimize the electrical contact between the moveable STM probe and the nanowire, which is a common challenge for characterizing the electrical properties of semiconductor nanostructures with a nanoprobe that needs to be addressed [27], [28],[31], [33]. The electron beam, with a diameter of a few nanometers, provided by the SEM is also utilized to study the characteristics of the built-in p-i-n junction in the individual nanowires by so called electron beam induced current (EBIC) measurements. The STM probe can also be used to apply strain on the nanowires. We find that the possibility to selectively choose and perform measurements on single nanowires in an ensemble of vertical nanowires offered by the STM probe inside the SEM is very useful to investigate the difference in performance between individual nanowires. These 
measurements allow the identification of the reason for the variation in performance, ultimately leading to suggestions on how to design nanowire solar cells for best performance.

\section{Materials and method}

We have used an STM-SEM sample holder fabricated by Nanofactory Instruments AB. The design of the STM part of the holder is illustrated in Figure 1 (a). The STM probe consists of a Au-wire with a diameter of $0.25 \mathrm{~mm}$. A sharp tip of the Au-wire can be created by cutting it at an approximate angle of $45^{\circ}$ with a pair of scissors while pulling the wire away from the scissors. The probe is attached to a probe holder which has six legs that are clamped around a movable conductive sphere. The movement of the sphere is controlled by sending electric signals to five piezoelectric plates that are positioned in a tubular fashion. This design enables two different movement modes of the probe, coarse and fine movement. Fine movement is achieved when the probe holder basically follows the movement of the sphere, and it provides an accuracy of $0.2 \AA$ in the forward/backward direction, and $2 \AA$ in the lateral and vertical directions for the probe tip position. Coarse movement is achieved when the sphere is moved by the piezo so fast that the inertia of the probe holder makes the legs slide across the sphere. This enables quick positioning of the probe with a range of several millimeters in all three dimensions. A more detailed description of the STM part of the holder is provided in [25]. The nanowire sample is mounted in front of the probe tip and the growth substrate is electrically connected to an external measurement circuit containing a picoammeter and a variable voltage supply, enabling I-V measurements. The probe is connected to the external circuit via the probe holder and the conductive sphere. A white LED was mounted on the STM-SEM holder to serve as the illumination source for the measurements involving light, see Figure S1 in Supplementary information. The holder with the LED is placed inside the 
vacuum chamber of a Zeiss Ultra 55 field emission gun SEM. An electric feed-through in the SEM enables the LED to be connected to a power supply and the STM to be connected to the operating system, comprising a pre-amplifier, a measurement/piezo control unit and a computer. The control unit contains the external measurement circuit described above and the electronics needed for sending the correct electrical signals to the piezo. The live image 
provided by the SEM and the well-controlled motion of the STM probe enable us to contact individual nanowires that are part of a nanowire ensemble.

(a)

Electron beam
in SEM
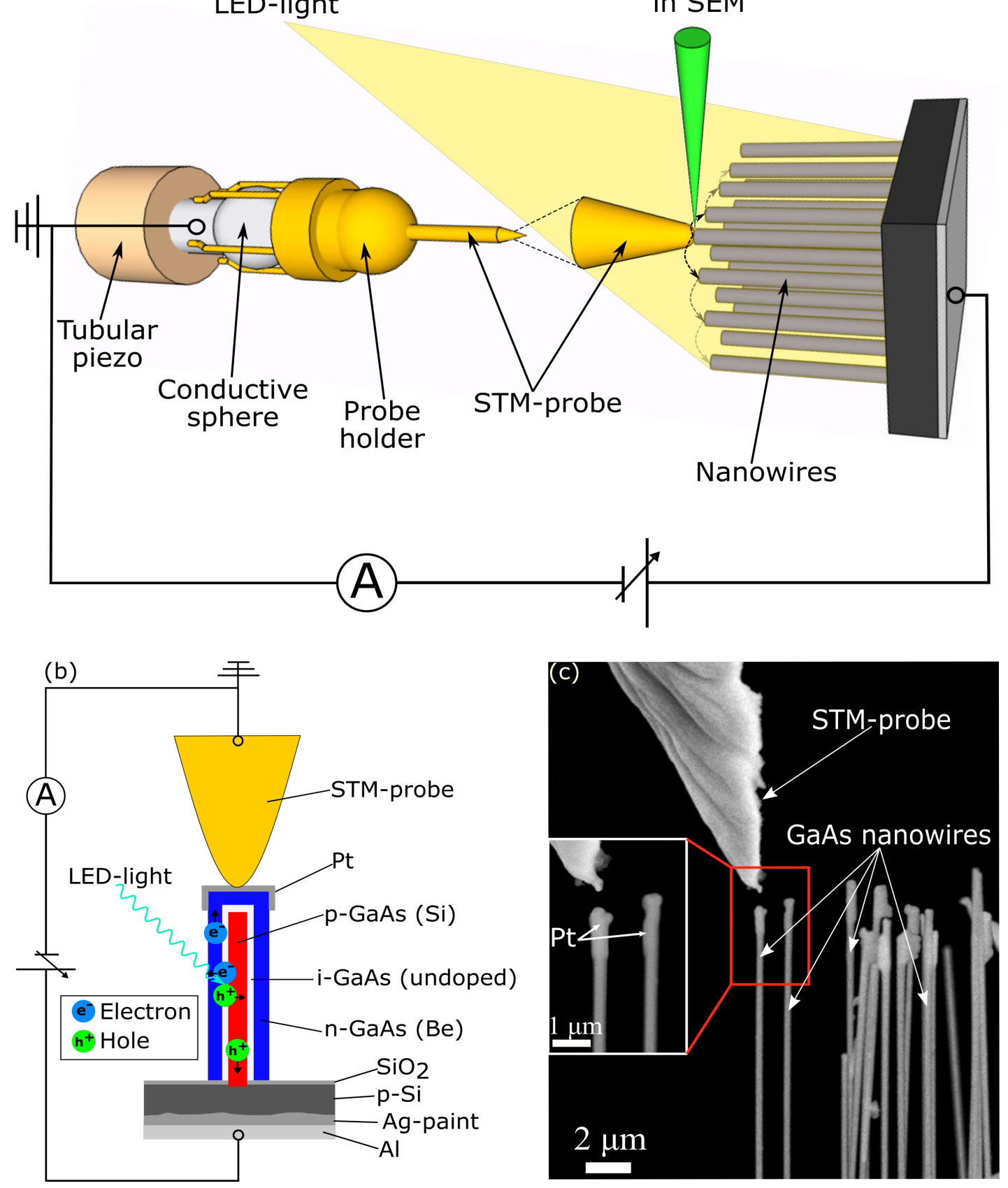

Figure 1 - Illustration of the STM-SEM technique. (a) The STM probe is brought into contact with individual nanowires by using piezoelectric motion in the SEM. An LED is used to illuminate the sample. The probe and the sample are connected to an external circuit containing a picoammeter and a variable voltage supply. (b) The STM probe is brought into contact with the tip of a nanowire, where a thin layer of Pt has been deposited. In this way, the n-doped region of the nanowire is contacted to the external circuit. The pdoped core of the nanowire is connected to the circuit via the p-doped Si substrate, a layer of Ag paint and an Al plate. When photons from the LED are absorbed in the nanowire, they generate electron-hole pairs that separate in the built-in field of the $p$-i- $n$ junction. This gives rise to a photocurrent in the external circuit. (c) SEM image of the STM probe close to a nanowire sample. The inset highlights the Pt deposition at the tip of the nanowires. 
The investigated GaAs nanowires were grown vertically on a p-doped Si substrate by a selfcatalyzed molecular beam epitaxy (MBE) method, further details are provided in [9]. The nanowires were $15-25 \mu \mathrm{m}$ long with a diameter of $250-350 \mathrm{~nm}$. They were doped to form a radial p-i-n junction within each wire, see Figure 1 (b). The figure also illustrates the setup for the electrical characterization. The STM probe is brought into contact with the ndoped shell of the nanowire. In order to increase the metal-semiconductor contact area, a thin layer of Pt is deposited on the tip of the nanowire using electron beam induced deposition in an FEI Versa3D focus ion beam instrument. The p-doped core of the nanowire is contacted through the p-doped $\mathrm{Si}$ substrate, $\mathrm{Ag}$ paint and an $\mathrm{Al}$ plate. A thin layer of $\mathrm{SiO}_{2}$ at the surface of the substrate prevents the n-doped layer to be in contact with the substrate. This in situ setup can be used to measure both the illuminated and the dark I-V characteristics of individual nanowires. When the LED is turned on, electron-hole pairs will be generated by the absorbed photons in the nanowire, and these charge carriers will be separated by the built-in field of the p-i-n junction, giving rise to a photocurrent. Figure 1 (c) shows an SEM micrograph of the STM probe and the nanowires. Please note the sharp tip of the mechanically cut Au-probe with a radius of approximately $120 \mathrm{~nm}$.

In order to ensure that the measurements represent the inherent properties of the nanowires, it is important that the contact resistance between the STM probe and the nanowire is low, so that the current is limited by the nanowire and not the contact resistance. As mentioned earlier we deposited a thin layer of Pt at the tip of the nanowire to increase the contact area, see the inset of Figure 1 (c). However, this is not enough. It is well-known that a Schottky barrier forms at the interface of a metal and GaAs, which may cause the contact to be currentrectifying depending on the width of the depletion layer. Since the nanowires have a relatively high doping concentration $\left(\sim 5 e^{18} \mathrm{~cm}^{-3}\right)$, the width of the depletion layer should be small enough for the charge carriers to tunnel through the barrier in both directions and make the 
contact practically ohmic [34]. However, the nanowires are covered with a thin native oxide layer, which has been shown to increase the resistance of a metal-GaAs contact [35].

Annealing has been successfully used to disperse the native oxide layer to improve the quality of metal-semiconductor contacts [36]. Therefore, we established a procedure to apply a $10 \mathrm{~V}$ bias between the STM probe and the sample to induce Joule heating, which improves the quality and reproducibility of the electrical contact significantly.

\section{Results and discussion}

\subsection{Photovoltaic measurements}

We measured the I-V characteristics of individual nanowires, both in dark and illuminated conditions. A few examples are shown in Figure 2. During the progress the I-V measurements, the electron beam was blanked. The dark I-V curves in Figure 2 (a) and (b) demonstrate a current-rectifying behavior, which is due to the p-i-n junction. The illuminated IV-curves are shifted downwards compared to the dark curves, due to the photocurrent induced by the LED photons. The quality of the metal-semiconductor contact is reflected in the illuminated I-V curves. Figure 2 (a) shows that the illuminated IV-curve has an S-shape around zero bias before the Joule heating process has been applied. It has been shown before that a rectifying contact in series with a p-n junction shows this S-shape characteristics [37]. Our annealing procedure of the contact resulted in the disappearance of the S-shape and an illuminated I-V curve corresponding to the dark I-V curve with a superimposed photocurrent [Figure $2(\mathrm{~b})$ ], indicating an ohmic contact with low resistance. One concern we have is that the Joule heating may cause harm to the p-i-n junction within the nanowire. However, in Figure 2 (b) it is clear that the junction remains after the Joule heating, indicating that the Joule heating only affected the contact between the Pt and the GaAs. 
The short circuit current $\left(\mathrm{I}_{\mathrm{SC}}\right)$, the open circuit voltage $\left(\mathrm{V}_{\mathrm{OC}}\right)$ and the fill factor $(\mathrm{FF})$ of the single nanowire can be extracted from the illuminated IV-curve, and their values are shown in Figure 2 (b). It is possible to obtain a value of the power conversion efficiency (PCE) of the single nanowire by using the formula $P C E=\frac{I_{S C} V_{O C} F F}{P_{\text {in }}}$, where $P_{i n}$ is the power of the photon flux reaching the projected area of the nanowire. Since the LED light is incident at an angle of approximately $40^{\circ}$ to the normal of the substrate, the projected area of the nanowire is $A_{p r}=$ Length $_{N W} *$ Diameter $_{N W} * \sin \left(40^{\circ}\right)$. The irradiance of the LED was determined, by use of a spectrometer, to be $6.3 \mathrm{mWcm}^{-2}$. The length and diameter of the nanowire were measured to be approximately $16 \mu \mathrm{m}$ and $250 \mathrm{~nm}$, respectively. The calculated efficiency of this nanowire is thus around $11 \%$. It should be noted that this value of the PCE is not directly comparable with measurements performed using a standard solar simulator, since the LED has a different intensity spectrum compared to a 1 sun illumination source (see Figure S2 in Supplementary information). Also, PCE values of single nanowires cannot be directly translated into PCE values of a corresponding full-scale nanowire array solar cell, because the projected area of the nanowire is not the same as the light absorbing area due to the waveguiding effect [1]. The PCE value can be used to compare the performance of single nanowires characterized under the same conditions though. In other words, we can use the PCE value to make a quantitative comparison between different nanowires.

Figure 2 (c) shows a comparison between the dark I-V curves of two nanowires, NW1 and NW2. It is evident that the two I-V curves differ substantially, with the most noticeable difference at around zero bias. While the curve of NW1 is nearly flat in this region, indicative of an ideal solar cell, the curve of NW2 has a relatively large gradient, indicative of a low shunt resistance which is detrimental for the performance of the cell. One possible reason for this would be if the n-doped shell and the intrinsic layer are deficient at the tip of NW2, allowing the Pt deposition to contact the p-doped core of the nanowire. This would result in a 
low shunt resistance. Since this work focuses on the development of the characterization method, we have not gone further in the investigation of the difference in performance between NW1 and NW2. 


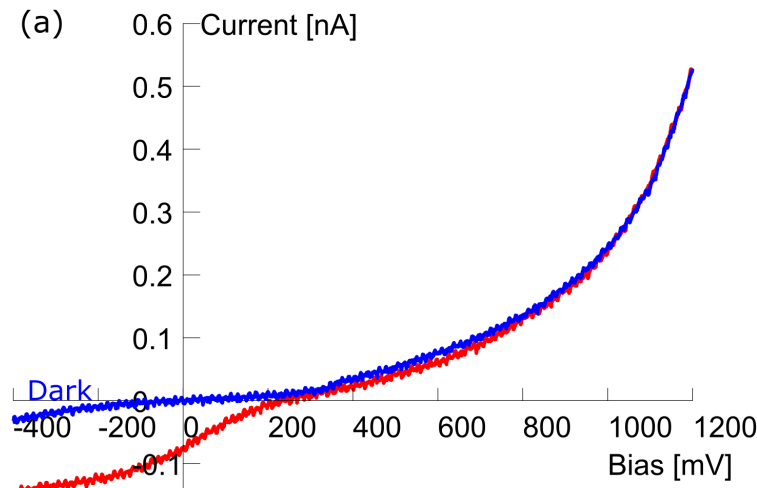

Tlluminated
-0.2

(b) 0.6 - Current $[\mathrm{nA}]$

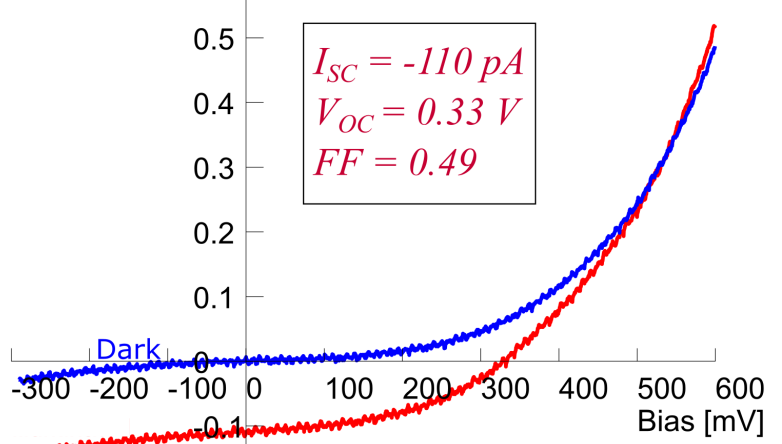

Illuminated

$-0.2$

(c)

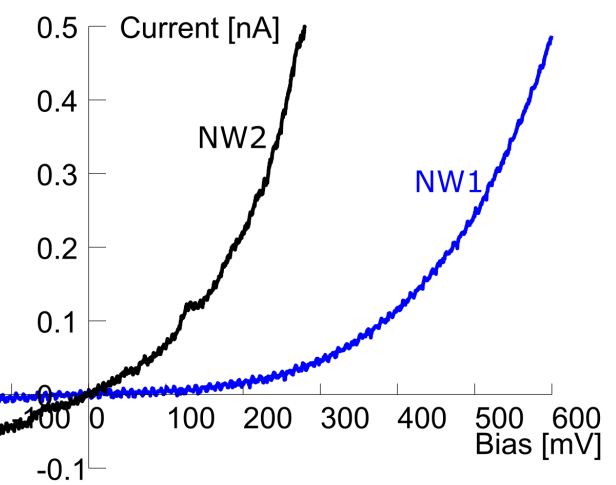

Figure $2-\mathrm{I}-\mathrm{V}$ measurements on single nanowires. All the measurements were performed without electron beam irradiation. (a) I-V characteristics in the dark and under LED illumination for NW1 before Joule heating. The illuminated I-V curve has an Sshape around zero bias, indicating a rectifying metalsemiconductor contact. (b) I-V characteristics in the dark and under LED illumination for NW1 after Joule heating. The S-shape is gone, indicating ohmic contact. The parameters short circuit current $\left(\mathrm{I}_{\mathrm{SC}}\right)$, open circuit voltage $\left(\mathrm{V}_{\mathrm{OC}}\right)$ and fill factor $(\mathrm{FF})$ are included. (c) Comparison of the dark I-V characteristics of NW1 and NW2. 
The mobile STM probe also provides the possibility to induce mechanical strain during the electrical measurements by bending the nanowires, see Error! Reference source not found. (a). A crude approximation of the amount of strain induced by the bending can be made using the relation $\varepsilon= \pm r / R$, where $\varepsilon$ is the strain, $r$ is the radius of the nanowire and $R$ is the radius of the curvature of the most bent part of the nanowire. Using this relation, we estimate the strain to be within the range of $\pm 0.5-1.5 \%$ in the most bent part of NW2. At the outer perimeter of the wire the strain will be tensile (positive), and at the inner perimeter it will be compressive (negative). Error! Reference source not found. (b) shows a comparison of the
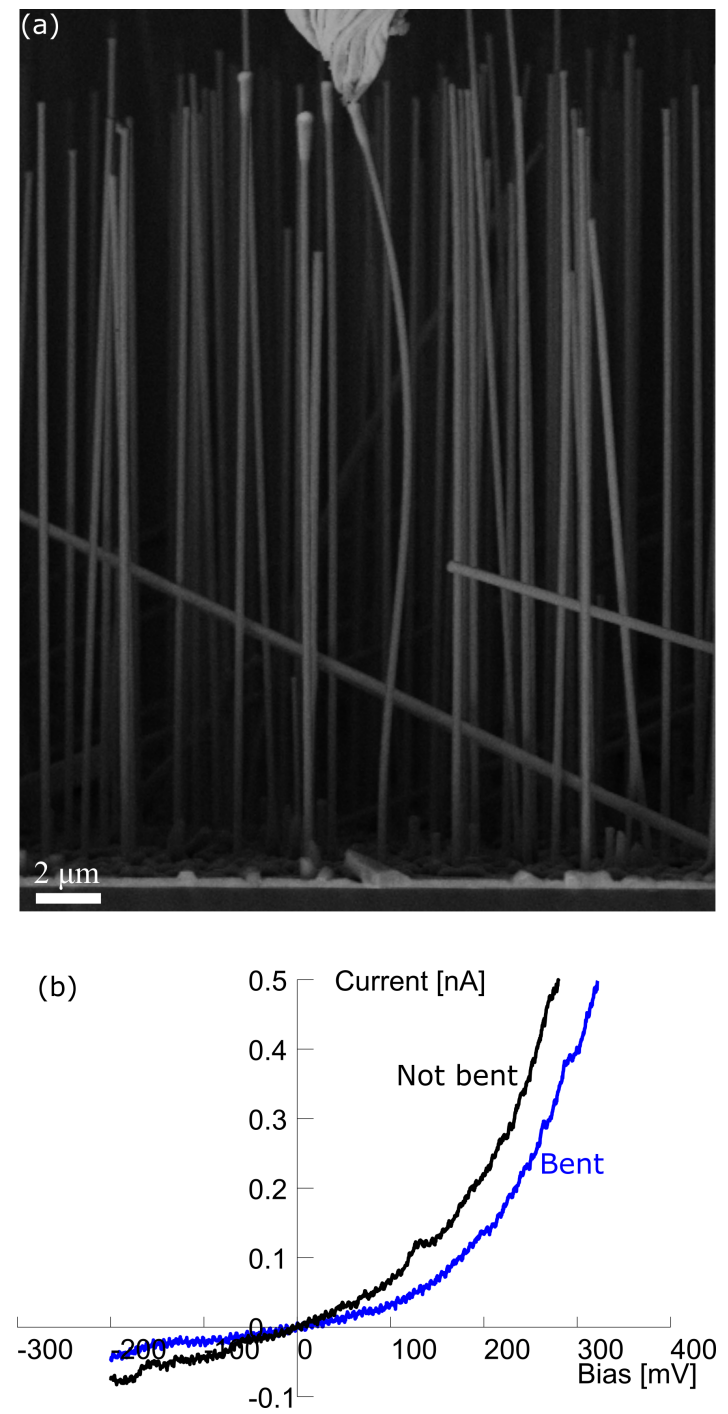

Figure 3 - (a) SEM image showing how the STM probe can be used to bend a nanowire. (b) Comparison of the I-V characteristic of NW2 when it was bent and unbent. The measurement was performed without electron beam irradiation.

dark I-V characteristics of NW2 with and without bending. The bending affects the I-V 
characteristics of the nanowire. Please see section S4 in Supplementary information for a discussion about this.

\subsection{EBIC measurements}

Since the measurements are performed inside an SEM, the electron beam can be used to generate electron-hole pairs in the nanowire resulting in an electron beam induced current (EBIC). The main difference between photocurrent measurements and EBIC measurements is that the LED illuminates the whole nanowire while the electron beam can be focused to a spot only a few nanometers wide, enabling local characterization of different parts of the nanowire. The spatial resolution of EBIC measurements is larger than the spot size of the beam though, due to the beam broadening occurring inside the sample. The EBIC resolution is around 200 $\mathrm{nm}$ for a $5 \mathrm{kV}$ acceleration voltage and around $250 \mathrm{~nm}$ for $20 \mathrm{kV}$, see Figure $\mathrm{S} 3$ in Supplementary information for a Monte Carlo simulation of the beam interaction volume. By scanning the nanowire with the electron beam while recording the EBIC signal, an EBIC map can be produced, see Figure 4. Here the SEM image of a nanowire [Figure 4 (a)] and the corresponding EBIC maps at a beam acceleration voltage of $20 \mathrm{kV}$ [Figure 4 (b)] and $5 \mathrm{kV}$ [Figure 4 (c)] are shown. There are a few aspects of the EBIC results that should be noted. First of all, the EBIC signal on the Au probe [upper left corner in Figure 4 (a), (b) and (c)] is much smaller than the EBIC signal on the nanowire. This is a verification that the nanowire contains a functioning $\mathrm{p}-\mathrm{i}-\mathrm{n}$ junction that effectively separates the generated electron-hole pairs. The explanation is that the EBIC signal on the Au probe is limited by the beam current 
value, which is roughly $1 \mathrm{nA}$, since no electron-hole pairs are generated in a metal. On the other hand, inside the semiconductor nanowire each beam electron could generate thousands of electron-hole pairs, since the average energy it takes to generate one electron-hole pair in GaAs by electron radiation, multiple scattering processes considered, is around $4.6 \mathrm{eV}$ [40]. As a result, the EBIC signal measured when the beam is focused on the nanowire reaches values as high as $72 \mathrm{nA}$. The second observation is that the EBIC signal is in general quite constant in the direction along the nanowire at both electron beam energies. This can be attributed to the radial junction geometry of the nanowire. The radial geometry ensures that the diffusion distance, for the minority carriers to reach the junction, is short no matter where the electron-hole pairs are created along the nanowire. Therefore, the charge separation efficiency is constant along the nanowire, as opposed to the situation in nanowires with axial junctions, where the charge separation efficiency depends strongly on the position along the nanowire (ref). The EBIC maps also show that the junction quality is high and homogeneous along the nanowire, without any obvious defective domains. The fact that the EBIC signal is significantly weaker at the edges of the nanowire than in the center is mainly due to that a greater part of the beam electrons escapes before the entire beam energy has been deposited at the edge of the nanowire. A last observation is that at the tip of the nanowire, the EBIC signal at a beam acceleration voltage of $20 \mathrm{kV}(5 \mathrm{kV})$ is slightly higher (lower) compared to the rest of the nanowire. In the case of $20 \mathrm{kV}$, this is probably because the electrons that are transmitted through the tip of nanowire will end up in the STM-probe and make a small additional contribution to the current. At $5 \mathrm{kV}$, the slightly lower EBIC signal at the tip of the 
nanowire is probably due to surface contamination, reducing the number of electrons reaching into the nanowire.

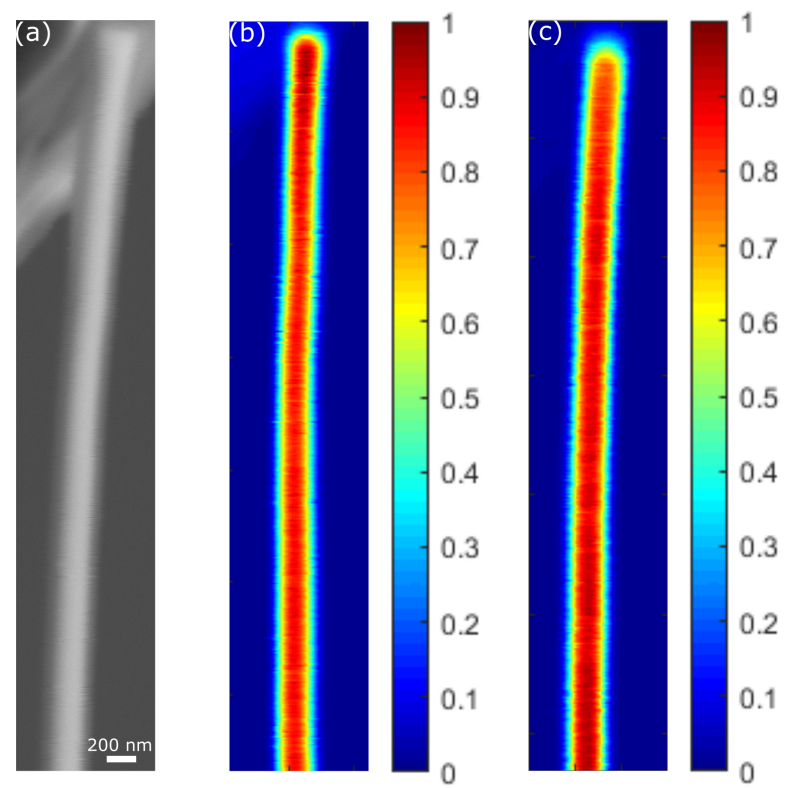

Figure 4 - (a) SEM micrograph of a nanowire contacted by the STM-probe and corresponding normalized EBIC maps at a beam acceleration voltage of (b) $20 \mathrm{kV}$ and (c) $5 \mathrm{kV}$.

\subsection{Evaluation of the technique}

The advantage of the STM-SEM technique compared to the existing techniques for characterizing single, photovoltaic nanowires discussed in the introduction of this paper, is that it can be used to directly compare the photovoltaic properties of different nanowires on as-grown substrates. There is no need to remove the nanowires from the growth substrate or to fabricate special single-wire cells. If a nanowire is found to perform badly, the SEM imaging can be used to find possible reasons for that, and it could lead to insights valuable for the development of the nanowire solar cell technology. Additionally, the possibility to bend the nanowires with the STM probe could be important for investigation of the possibility to use elastic strain engineering [41] to enhance the performance of the solar cells. The technique is also well suited for EBIC measurements. A few drawbacks of the technique have been identified. The limited space inside the SEM chamber obstructs the use of a standard 
solar simulator to illuminate the nanowires, which would be preferable to the LED in order to be able to compare the PCE values to other studies. A possible solution to achieve simulated sunlight inside the SEM would be to introduce an optical fiber into the chamber. Another way would be to use a compact solar simulator consisting of a combination of LED:s [42]. Another drawback is that the STM probe may block light that is incident parallel to the nanowires. Our solution was to mount the LED so that the light was incident at an angle of $40^{\circ}$ to the normal of the substrate surface. This could affect the performance of the nanowires because the waveguiding effect is mostly pronounced when the light is incident parallel to the long axis of the nanowires. In principle, it should be possible for the STM probe to contact the side of a nanowire to avoid the parallel light to be blocked.

\section{Conclusion}

A technique for investigating photovoltaic and electrical properties of single, vertical semiconductor nanowires on as-grown substrates utilizing an STM-SEM sample holder and a LED light source was developed. The functionality of the technique was demonstrated by performing dark and illuminated IV-measurements, as well as EBIC measurements, on GaAs nanowires with radial p-i-n junctions grown on a p-doped Si substrate. The technique enables the comparison of the performance of single nanowires, that are part of a nanowire ensemble, and correlate it to the nanowire structure. It is also possible to apply strain to the nanowires while performing measurements. These abilities are not available in the most common existing techniques for characterizing single photovoltaic nanowires. Therefore, it provides useful information for understanding the effects of microscopic structure on the photovoltaic properties and improving the performance of nanowire solar cells. 


\section{Acknowledgements}

JH, LJZ, LDK and EO acknowledge the financial support from Swedish Research Council (VR) under Grant No. 2016-04618 and the Nanoscience and Nanotechnology Area of Advance and Energy Area of Advance at Chalmers University of Technology. JH and LJZ would also like to thank Steven Jones for the help on the measurement of the spectral irradiance of the LED illumination source.

\section{Declaration of interest}

The authors declare no competing financial interests.

\section{Appendix A. Supplementary information}

\section{References}

[1] G. Otnes and M. T. Borgström, "Towards high efficiency nanowire solar cells," Nano Today, vol. 12, pp. 31-45, 2017.

[2] E. C. Garnett, M. L. Brongersma, Y. Cui, and M. D. Mcgehee, "Nanowire Solar Cells," Annu. Rev. Mater. Res., vol. 41, pp. 269-295, 2011.

[3] R. R. Lapierre et al., "III-V nanowire photovoltaics: Review of design for high efficiency," Phys. Status Solidi - Rapid Res. Lett., vol. 7, no. 10, pp. 815-830, 2013.

[4] S. Mokkapati and C. Jagadish, "Review on photonic properties of nanowires for photovoltaics," Opt. Express, vol. 24, no. 15, pp. 17345-17358, 2016. 
[5] D. Van Dam et al., "High-Efficiency Nanowire Solar Cells with Omnidirectionally Enhanced Absorption Due to Self-Aligned Indium-Tin-Oxide Mie Scatterers," ACS Nano, vol. 10, no. 12, pp. 11414-11419, 2016.

[6] J. Kupec, R. L. Stoop, and B. Witzigmann, "Light absorption and emission in nanowire array solar cells," Opt. Express, vol. 18, no. 26, pp. 27589-27605, 2010.

[7] C. Lin and M. L. Povinelli, "Optical absorption enhancement in silicon nanowire arrays with a large lattice constant for photovoltaic applications," Opt. Express, vol. 17, no. 22, pp. 19371-19381, 2009.

[8] J. Wallentin et al., "InP nanowire array solar cells achieving 13.8\% efficiency by exceeding the ray optics limit.," Science, vol. 339, no. 6123, pp. 1057-1060, 2013.

[9] P. Krogstrup et al., "Single-nanowire solar cells beyond the Shockley-Queisser limit," Nat. Photonics, vol. 7, pp. 306-310, 2013.

[10] N. Anttu and H. Q. Xu, "Efficient light management in vertical nanowire arrays for photovoltaics.," Opt. Express, vol. 21, no. S3, pp. A558-A575, 2013.

[11] C. Lin and M. L. Povinelli, "Optical absorption enhancement in silicon nanowire arrays with a large lattice constant for photovoltaic applications.," Opt. Express, vol. 17, no. 22, pp. 19371-81, 2009.

[12] C. H. Henry, "Limiting efficiencies of ideal single and multiple energy gap terrestrial solar cells," J. Appl. Phys., vol. 51, no. 8, pp. 4494-4500, 1980.

[13] E. Ertekin, P. A. Greaney, D. C. Chrzan, and T. D. Sands, "Equilibrium limits of coherency in strained nanowire heterostructures," J. Appl. Phys., vol. 97, no. 11, 2005.

[14] T. Mårtensson et al., "Epitaxial III - V Nanowires on Silicon,” Nano Lett., vol. 4, no. 10, pp. 1987-1990, 2004. 
[15] B. M. Kayes, H. A. Atwater, and N. S. Lewis, "Comparison of the device physics principles of planar and radial p-n junction nanorod solar cells," J. Appl. Phys., vol. 97, no. 11,2005 .

[16] M. C. Beard, J. M. Luther, and A. J. Nozik, "The promise and challenge of nanostructured solar cells," Nat. Nanotechnol., vol. 9, no. 12, pp. 951-954, 2014.

[17] C. Gutsche et al., "Direct determination of minority carrier diffusion lengths at axial GaAs nanowire p-n junctions," Nano Lett., vol. 12, no. 3, pp. 1453-1458, 2012.

[18] S.A. Mann, S. Z. Oener, A. Cavalli, J. E. M. Haverkort, E. P. A. M. Bakkers, and E. C. Garnett, "Quantifying losses and thermodynamic limits in nanophotonic solar cells," Nat. Nanotechnol., vol. 11, no. 12, pp. 1071-1075, 2016.

[19] B. Tian et al., "Coaxial silicon nanowires as solar cells and nanoelectronic power sources," Nature, vol. 449, pp. 885-889, 2007.

[20] C. Colombo, M. Heiß, M. Grätzel, and A. Fontcuberta I Morral, "Gallium arsenide p-in radial structures for photovoltaic applications," Appl. Phys. Lett., vol. 94, no. 17, 2009.

[21] A. Nowzari et al., "A comparative study of absorption in vertically and laterally oriented InP core-shell nanowire photovoltaic devices," Nano Lett., vol. 15, no. 3, pp. $1809-1814,2015$.

[22] M. Heiss and A. Fontcuberta I Morral, "Fundamental limits in the external quantum efficiency of single nanowire solar cells," Appl. Phys. Lett., vol. 99, no. 26, pp. 97-100, 2011.

[23] G. Otnes et al., "Understanding InP Nanowire Array Solar Cell Performance by Nanoprobe-Enabled Single Nanowire Measurements," Nano Lett., vol. 18, no. 5, pp. 
3038-3046, 2018.

[24] D. Mikulik et al., "Conductive-probe atomic force microscopy as a characterization tool for nanowire-based solar cells," Nano Energy, vol. 41, pp. 566-572, 2017.

[25] K. Svensson, Y. Jompol, H. Olin, and E. Olsson, "Compact design of a transmission electron microscope-scanning tunneling microscope holder with three-dimensional coarse motion," Rev. Sci. Instrum., vol. 74, no. 11, pp. 4945-4947, 2003.

[26] Y. Oshima, K. Mouri, H. Hirayama, and K. Takayanagi, "Development of a miniature STM holder for study of electronic conductance of metal nanowires in UHV-TEM," Surf. Sci., vol. 531, no. 3, pp. 209-216, 2003.

[27] M. Berthe, C. Durand, T. Xu, J. P. Nys, P. Caroff, and B. Grandidier, "Combined STM and Four-Probe Resistivity Measurements on Single Semiconductor Nanowires," At. Scale Interconnect. Mach., pp. 107-118, 2012.

[28] C. Durand et al., "Electrical characterization of semiconductor nanowires by scanning tunneling microscopy,” Proc. SPIE, vol. 8996, p. 89960E1-89960E10, 2014.

[29] F. Léonard, A. A. Talin, B. S. Swartzentruber, and S. T. Picraux, "Diameter-Dependent Electronic Transport Properties of Au-Catalyst/Ge-Nanowire Schottky Diodes,” Phys. Rev. Lett., vol. 102, pp. 1-4, 2009.

[30] S. Korte et al., "Resistance and dopant profiling along freestanding GaAs nanowires," Appl. Phys. Lett., vol. 103, no. 14, 2013.

[31] M. W. Larsson, L. R. Wallenberg, A. I. Persson, and L. Samuelson, "Probing of individual semiconductor nanowhiskers by TEM-STM," Microsc. Microanal., vol. 10, no. 1, pp. 41-46, 2004.

[32] A. A. Talin, B. S. Swartzentruber, F. Leonard, X. Wang, and S. D. Hersee, "Electrical 
transport in GaN nanowires grown by selective epitaxy," J. Vac. Sci. Technol. B, vol. 27, p. 2040, 2009.

[33] Y.-P. Zeng, Y.-G. Wang, B.-H. Qu, and H.-C. Yu, "Cathode-Control Alloying at an Au-ZnSe Nanowire Contact via in Situ Joule Heating," Chinese Phys. Lett., vol. 29, no. 8, p. $88105,2012$.

[34] V. L. Rideout, “A review of the theory and technology for ohmic contacts to group IIIV compound semiconductors," Solid. State. Electron., vol. 18, no. 6, pp. 541-550, 1975.

[35] S. Ashok, J. M. Borrego, and R. J. Gutmann, "Electrical characteristics of GaAs MIS Schottky diodes," Solid State Electron., vol. 22, no. 7, pp. 621-631, 1979.

[36] A. Piotrowska, "Ohmic Contacts to GaAs: Fundamentals and Practice," Acta Phys. Pol. $A$, vol. 84, no. 3, pp. 491-504, 1993.

[37] Z. Zekry and G. Eldallal, "Effect of MS contact on the electrical behaviour of solar cells," Solid State Electron., vol. 31, no. 1, pp. 91-97, 1988.

[38] J. Greil, A. Lugstein, C. Zeiner, G. Strasser, and E. Bertagnolli, “Tuning the electrooptical properties of germanium nanowires by tensile strain," Nano Lett., vol. 12, no. 12, pp. 6230-6234, 2012.

[39] N. Healy et al., "Extreme electronic bandgap modification in laser-crystallized silicon optical fibres," Nat. Mater., vol. 13, no. 12, pp. 1122-1127, 2014.

[40] C. J. Wu and D. B. Wittry, "Investigation of minority-carrier diffusion lengths by electron bombardment of Schottky barriers," J. Appl. Phys., vol. 49, no. 5, pp. 28272836, 1978.

[41] J. Li, Z. Shan, and E. Ma, "Elastic strain engineering for unprecedented materials 
properties," MRS Bull., vol. 39, no. 2, pp. 108-114, 2014.

[42] A. Novickovas, A. Baguckis, A. Mekys, and V. Tamosiunas, "Compact light-emitting diode-based AAA class solar simulator: Design and application peculiarities," IEEE J. Photovoltaics, vol. 5, no. 4, pp. 1137-1142, 2015. 


\section{Supplementary information}

\section{An STM - SEM Setup for Characterizing Photon and Electron Induced Effects in Single Photovoltaic Nanowires}

Jonatan Holmér ${ }^{1}$, Lunjie Zeng ${ }^{1}$, Thomas Kanne ${ }^{2}$, Peter Krogstrup ${ }^{2}$, Jesper Nygård $^{2}$, Ludvig de Knoop ${ }^{1}$, Eva Olsson ${ }^{1}$

${ }^{I}$ Department of Physics, Chalmers University of Technology, 41296, Göteborg, Sweden.

${ }^{2}$ Center for Quantum Devices and Station Q Copenhagen, Niels Bohr Institute, University of Copenhagen, Universitetsparken 5, 2100 Copenhagen, Denmark.

\section{S1 The STM-SEM holder}

The STM-SEM holder that was used for the experiments is shown in Figure S1. The LED is mounted on the side of the holder, and the angle of incidence of the light is approximately $40^{\circ}$ to the normal of the growth substrate of the nanowires. 


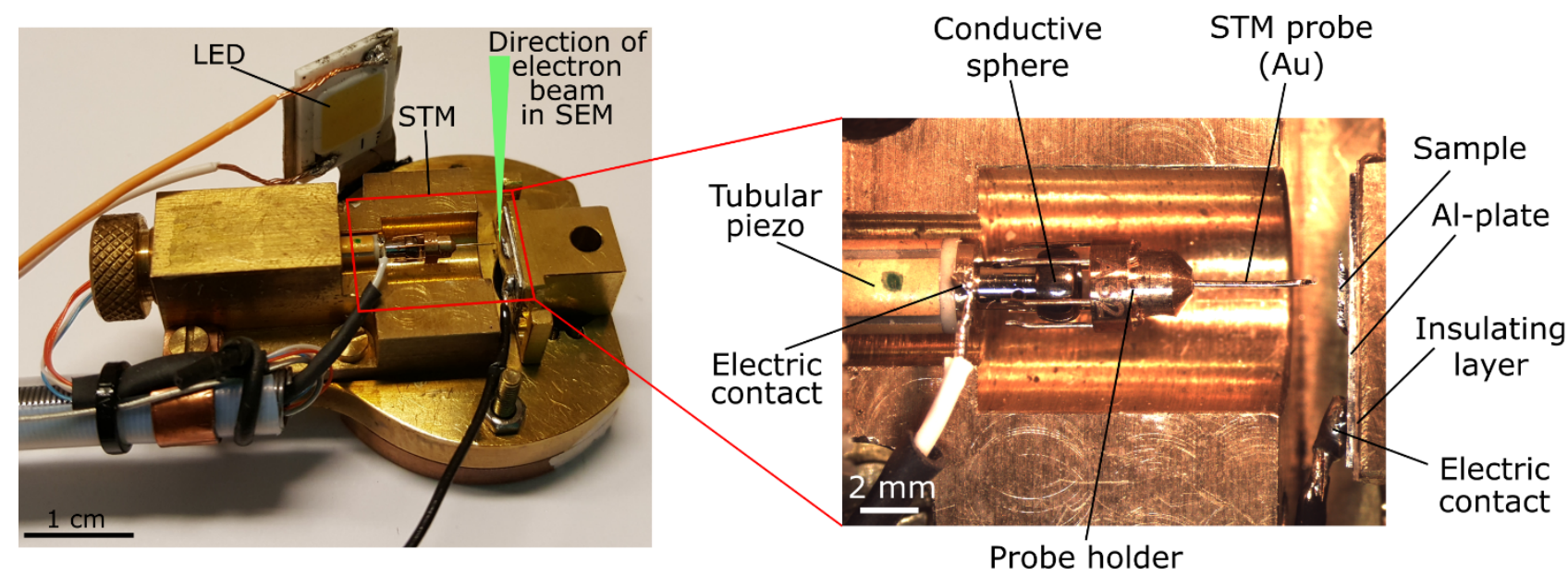

Figure S5 - Photograph of the STM-SEM holder. The LED is mounted on the side of the holder. The inset shows a zoomed view of the STM part of the holder.

\section{S2 The LED spectrum}

Figure S2 shows a comparison between the spectral irradiance of the LED and AM1.5

sunlight [1]. The fact that a part of the sunlight spectrum corresponds to photon energies

lower than the band gap energy of GaAs will make the efficiency of the nanowires at 1 sun

illumination lower compared to the efficiency at LED illumination. 


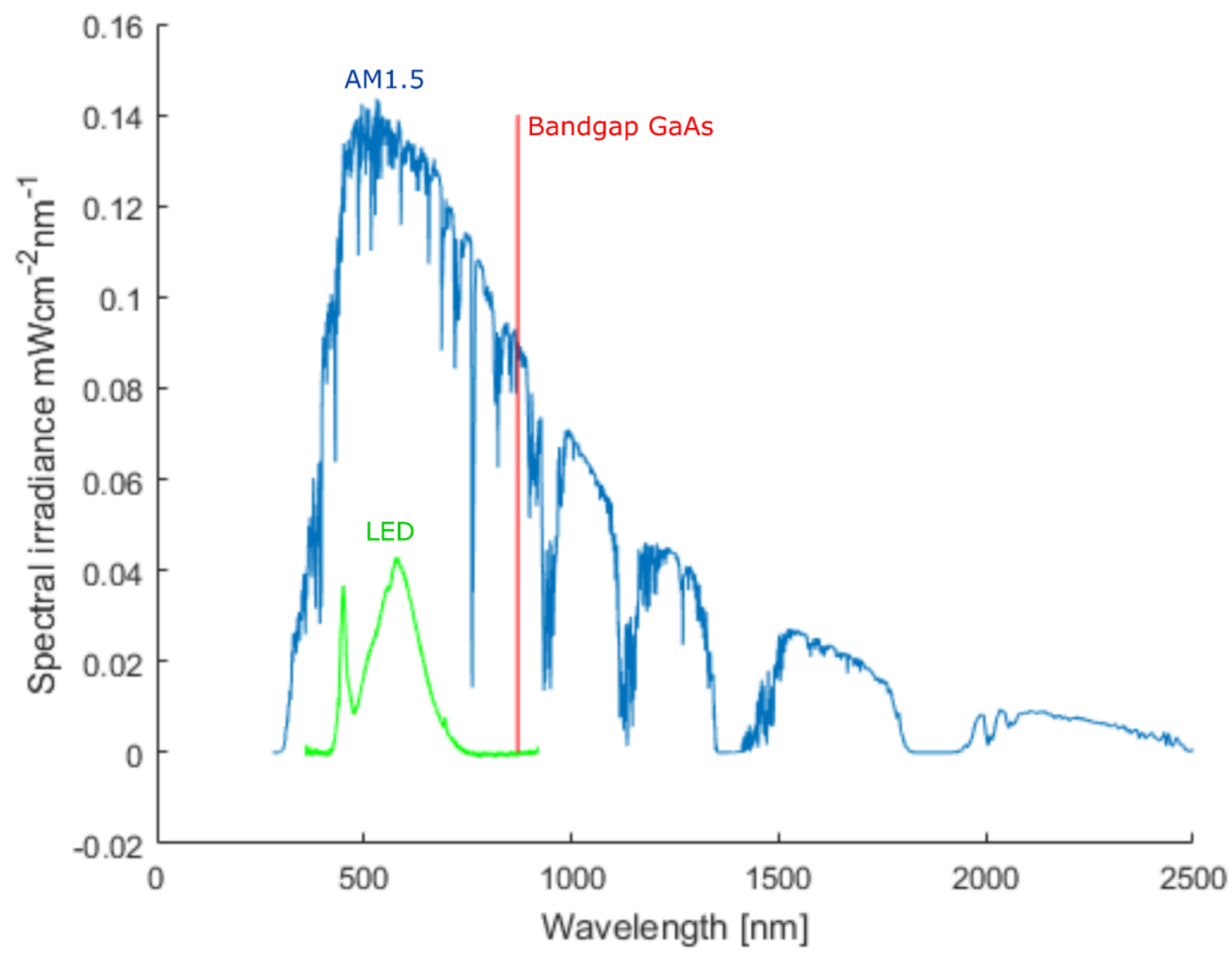

Figure S6 - Spectral irradiance of AM1.5 sunlight and the LED used for photocurrent measurements. The wavelength corresponding to the band gap energy of GaAs is indicated.

\section{S3 EBIC resolution}

Figure S3 shows Monte Carlo simulations of the electron beam interaction volume performed by using the Casino v3.3 software [2]. At a beam energy of $5 \mathrm{keV}$ the interaction volume diameter, and thereby the EBIC resolution, is around $200 \mathrm{~nm}$. At $20 \mathrm{keV}$, the interaction volume around $250 \mathrm{~nm}$ and part of the beam is transmitted through the nanowire. 

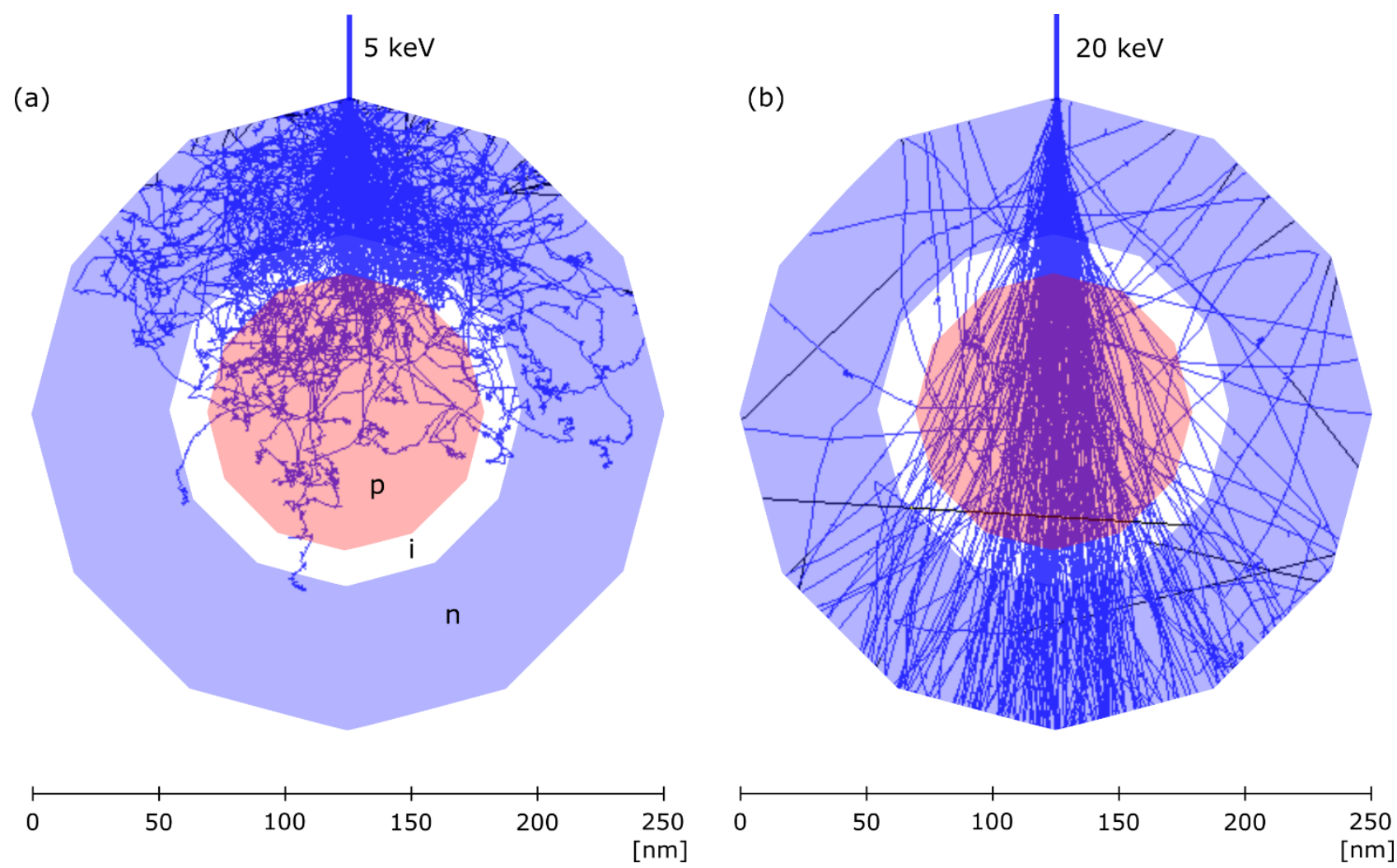

Figure S7 - Monte Carlo simulations of the beam interaction volume in a GaAs nanowire at beam acceleration voltages of (a) $5 \mathrm{kV}$ and (b) $20 \mathrm{kV}$. The simulations were performed using the Casino v3.3 software [2].

\section{S4 Bending nanowire}

When the nanowire is bent, there will be large tensile strain at the outer perimeter of the wire and large compressive strain at the inner perimeter, compared to in the center of the wire. This means that the strain will be larger in the n-doped region than in the pdoped region. Previous research has shown that both compressive and tensile strain reduces the band gap energy of GaAs. The bending of the nanowire should therefore lead to a transition from a $\mathrm{p}-\mathrm{i}-\mathrm{n}$ homojunction to a $\mathrm{p}-\mathrm{i}-\mathrm{n}$ heterojunction in the nanowire, and we expect this to affect the I-V characteristics. However, a change in band-gap energy alone does not tell us how the I-V characteristics are affected by the bending. We must also know how the work function is affected. Unfortunately, to our knowledge there has been no studies of the effect of strain on the work function of 
GaAs. One possible option is that the work function of the strained n-layer decreases.

The situation would then be similar to the one depicted in Figure S4 (c). The strength of the total built-in field of this $\mathrm{p}-\mathrm{n}$ heterojunction is larger than in the $\mathrm{p}-\mathrm{n}$ homojunction illustrated in Figure $\mathrm{S} 4(\mathrm{~b})$, since $\mathrm{qV}_{\mathrm{bi}}=\mathrm{E}_{\mathrm{f}, \mathrm{p}}-\mathrm{E}_{\mathrm{f}, \mathrm{n}}$. A stronger built-in field $\mathrm{V}_{\mathrm{bi}}$ should result in a higher $\mathrm{V}_{\mathrm{OC}}$. Therefore, this might be a possible explanation for the observed change in I-V characteristics when bending the nanowire, because looking at Figure 3 the I-V curve of the bent nanowire seems to result in a higher $\mathrm{V}_{\mathrm{OC}}$, assuming that the illuminated I-V curve is a superposition of the dark I-V curve.

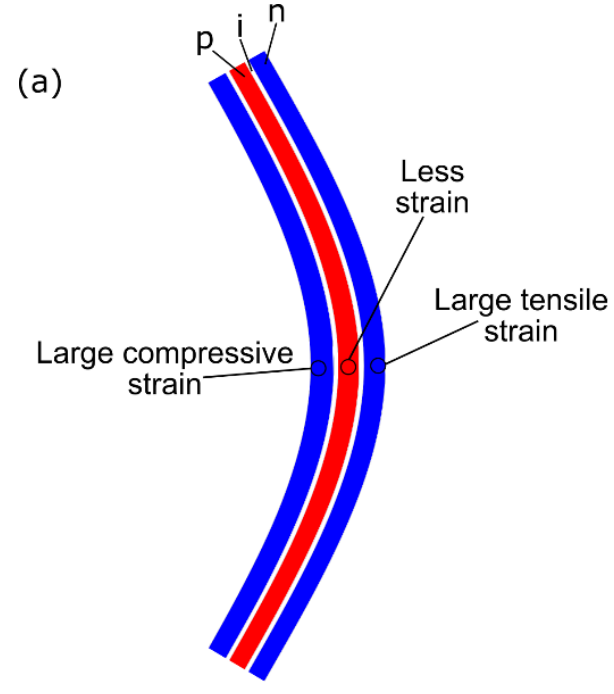

(b)

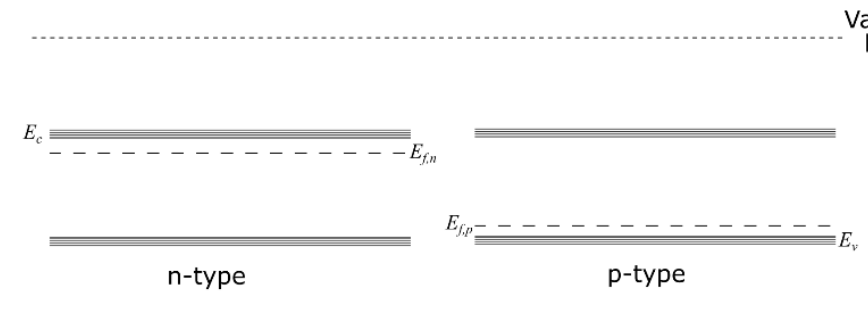

(c)

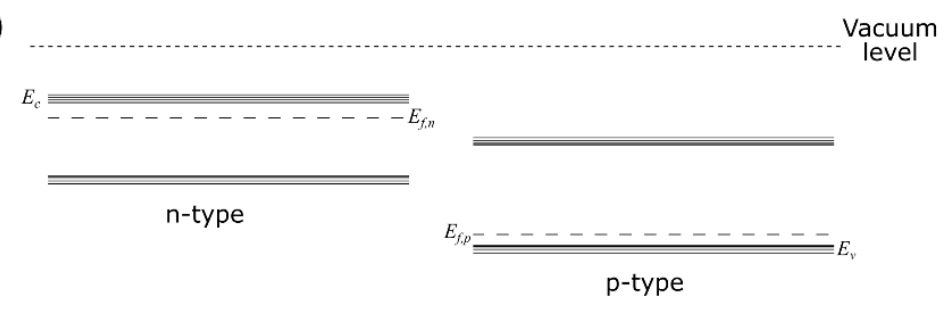

Figure S8 - When the nanowire is bent, the strain will be larger in the n-doped shell.

\section{References}

[1] ASTM G173-03(2012), "Standard Tables for Reference Solar Spectral Irradiances: Direct Normal and Hemispherical on $37^{\circ}$ Tilted Surface," ASTM International, West Conshohocken, PA, 2012, https://doi.org/10.1520/G0173-03R12 
[2] Demers, Hendrix et al. "Three-Dimensional Electron Microscopy Simulation with the CASINO Monte Carlo Software.” Scanning 33.3 (2011): 135-146. PMC. Web. 26 Mar. 2018. 